\title{
Atividade física como fator de proteção para comorbidades cardiovasculares em mulheres obesas
}

\author{
Physical activity as a protective factor against cardiovascular
} comorbidities in obese women

\author{
Cristiano Penas Seara Pitanga \\ Ricardo Jacó de Oliveira \\ Ines Lessa \\ Maria Cecília Costa \\ Francisco José Gondim Pitanga
}

1 União Metropolitana de Educação e Cultura, Lauro de Freitas, BA.

Brasil.

2 Universidade Católica de Brasília. Brasília, DF. Brasil.

3 Universidade Federal da Bahia. Salvador, BA. Brasil.

Recebido em 02/10/09 Revisado em 06/11/09 Aprovado em 23/04/10
Resumo - O objetivo do estudo foi analisar o efeito protetor da atividade física (AF) para comorbidades cardiovasculares (CMCV) em mulheres obesas. O estudo foi transversal, utilizando amostra composta por 387 mulheres obesas com índice de massa corporal (IMC) $\geq 30 \mathrm{~kg} / \mathrm{m}^{2}$. Utilizou-se a regressão logística para estimar a odds ratio (OR) com intervalo de confiança (IC) de 95\% e avaliar a associação entre AF e CMCV. Os dados foram analisados através do STATA, versão 7.0. Após ajustamento para idade, a AF apresentou associação com as CMCV: $\mathrm{OR}=0,11(0,04-0,25)$. Após estratificação por etnia, estado civil e IMC, as associações continuaram significativas. Foi também analisado o efeito dose resposta, sendo observado que as mulheres muito ativas apresentaram maior proteção contra as CMCV. A AF deve ser sugerida para mulheres obesas visando evitar as CMCV.

Palavras-chave: Atividade Física; Obesidade; Comorbidades; Cardiovasculares.

Abstract - The objective of this study was to analyze the protective effect of physical activity (PA) on cardiovascular comorbidities (CC) in obese women. A cross-sectional study was conducted on 387 obese women with a body mass index (BMI) $\geq 30 \mathrm{~kg} / \mathrm{m}^{2}$. Logistic regression analysis was used to estimate odds ratios (OR) with a 95\% confidence interval and to evaluate the association between PA and CC. Data were analyzed using the Stata 7.0 program. After adjustment for age, PA was associated with CC [OR $=0.11$ (0.04-0.25)]. This association continued to be significant after stratification by ethnic origin, marital status and BMI. Analysis of the dose-response effect showed a more marked protective effect on CC for very active women. PA should be recommended for obese women in order to prevent CC.

Key words: Physical activity; Obesity; Cardiovascular comorbidities. 


\section{INTRODUÇÃO}

De acordo com as mais recentes estimativas globais, a partir de estudo em 106 países, em 2005, havia 937 milhões de pessoas com sobrepeso e 396 milhões de pessoas obesas no mundo. As projeções para 2030 estimam em 2,16 bilhões de pessoas com sobrepeso e 1,12 bilhões com obesidade e comorbidades associadas. 1 .

No Brasil, de acordo com dados do Instituto Brasileiro de Geografia e Estatística (IBGE), a obesidade atinge $11 \%$ da população, correspondendo a 10,5 milhões de pessoas ${ }^{2}$. Estudos de caráter epidemiológico têm demonstrado que a obesidade está associada com morbidade e mortalidade cardiovascular ${ }^{3,4}$. Outrossim, estudos sugerem que a atividade física proporciona significativa melhoria da função cardíaca de mulheres climatéricas ${ }^{5}$, com importante redução do risco de doenças cardiovasculares, com impactos significativos em todas as causas de mortalidade ${ }^{6}$. A atividade física tem demonstrado uma relação inversa com a morbidade e mortalidade cardiovascular, como também altos níveis de atividade física no tempo livre estão associados ao aumento da longevidade? Recentes $^{2}$ trabalhos têm verificado que a prática regular da atividade física está associada a menores índices de mortalidade em obesos ativos, quando comparados a obesos sedentários ${ }^{8}$.

Por outro lado, já há algum tempo, tem-se verificado a existência de sub-grupo de obesos não expostos às comorbidades cardiovasculares (CMCV), normalmente encontradas em pessoas com diagnóstico de obesidade como, por exemplo: hipertensão arterial, diabetes mellitus e dislipidemias, especulando-se que a atividade física poderia exercer importante papel na prevenção destes agravos metabólicos e cardiovasculares em indivíduos obesos?.

Contudo, ao se tentar verificar qual seria a associação da atividade física com as CMCV em mulheres obesas, verifica-se que existe uma escassez de estudos que avaliam esta relação. Assim, o objetivo do presente estudo foi analisar o efeito protetor da atividade física para as $\mathrm{CMCV}$ em mulheres obesas.

\section{PROCEDIMENTOS METODOLÓGICOS}

\section{Amostra}

Participaram do estudo 387 mulheres, residentes da cidade de Salvador Bahia, com faixa etária de 21 a 75 anos, com índice de massa corporal
(IMC) $\geq 30 \mathrm{~kg} / \mathrm{m}^{2}$, que estavam em tratamento para obesidade, matriculadas no ambulatório de obesidade do Hospital Universitário professor Edgar Santos (HUPES) da Universidade Federal da Bahia (UFBA) e no Centro de Referência Estadual para Assistência a Diabetes e Endocrinologia (CEDEBA) da Secretaria de Saúde do Estado da Bahia (SESAB) na cidade de Salvador- Bahia. O estudo foi aprovado pelo Comitê de ética do conselho Regional de Medicina do Estado da Bahia, parecer/ resolução no 110/2005.

Os voluntários foram esclarecidos sobre o estudo, sendo que todos assinaram termo de autorização de acordo com a resolução 196/1996 do Conselho Nacional de Saúde, em concordância com os princípios éticos contidos na Declaração de Helsinki (1964 reformulada em 2000), do World Medical Association.

\section{Variáveis de estudo}

Foram estudadas as seguintes variáveis: nível de atividade física (variável independente), $\mathrm{CMCV}$, representada pela hipertensão arterial, diabetes mellitus e dislipidemias (variável dependente), etnia, estado civil e IMC (covariáveis).

\section{Coleta de dados}

Os dados foram coletados por uma equipe de estudantes de medicina da UFBA que foram devidamente treinados para executar os seguintes protocolos: o IMC foi determinado pela divisão da massa corporal pela estatura ${ }^{2}$, sendo a massa corporal expressa em quilogramas $(\mathrm{kg})$ e a estatura, em metros (m). Adotou-se como critério ${ }^{10}$ para obesidade o valor $\geq 30 \mathrm{~kg} / \mathrm{m}^{2}$.

Para aferição da estatura, foi empregado um estadiômetro vertical da própria balança, com 210 $\mathrm{cm}$ de comprimento e escala de $0,1 \mathrm{~cm}$, e para a avaliação da massa corporal foi utilizada uma balança de plataforma (Filizola, Brasil), calibrada, graduada de zero a $150 \mathrm{~kg}$ e com precisão de $0,1 \mathrm{~kg}$.

Para determinação dos níveis de atividade física, foi utilizado o International Physical Activity Questionnaire (IPAQ), na versão curta, com aplicação de entrevista referente à semana anterior, contendo perguntas em relação à frequência e duração das atividades físicas moderadas, vigorosas e da caminhada ${ }^{11}$. Inicialmente, os participantes do estudo foram classificados como sedentários, insuficientemente ativos, ativos e muito ativos.

Os indivíduos classificados como sedentários foram aqueles que não realizaram nenhuma atividade física por, pelo menos, 10 minutos contínuos 
durante a semana. Os classificados como insuficientemente ativos foram aqueles que realizavam atividades físicas por, pelo menos, 10 minutos por semana, porém em quantidade insuficiente para serem classificados como ativos. Foram classificados como ativos aqueles que cumpriram as recomendações de praticar atividades físicas leves ou moderadas por 5 dias ou mais por semana e acumular 150 minutos de atividades físicas por semana; e/ou praticar atividades físicas vigorosas $\geq 3$ dias por semana e $\geq 20$ minutos por sessão. Os indivíduos classificados como muito ativos foram aqueles que cumpriram com as recomendações de praticar atividades vigorosas $\geq 5$ dias por semana $\geq$ que 30 minutos por sessão; ou praticar atividades vigorosas $\geq 3$ dias por semana e $\geq 20$ minutos por sessão, somado a prática de atividades leves e/ou moderadas $\geq 5$ dias por semana e $\geq 30$ minutos por sessão. Em seguida, a classificação da atividade física foi dicotomizada em inativos fisicamente (sedentários e insuficientemente ativos) e ativos fisicamente (ativos e muito ativos).

Para determinação dos níveis de lipídios plasmáticos e glicemia, foi realizada coleta de sangue após 12 horas de jejum, no laboratório de Patologia Clínica (LPC), na cidade de Salvador-Bahia. Para a determinação dos níveis de HDL-C, foi utilizado o método "COD-ANA enzimático", enquanto que para determinação dos níveis de colesterol e triglicerídios, utilizou-se o método "enzimatico trinder". Os níveis de LDL-C e VLDL-C foram calculados de acordo com a fórmula de Friedewald. Para a caracterização do quadro de dislipidemia, foi adotado o critério de apresentar, pelo menos, uma das lípidas com o valor fora do limite recomendado pelas diretrizes Brasileiras para Dislipidemia da Sociedade Brasileira de Cardiologia ${ }^{12}$. Foram incluídos, também, os indivíduos dislipidêmicos em tratamento atual com medicamentos.

Para a determinação dos níveis de glicose sanguínea, foi utilizado o método "Oxidase GODANA enzimático". Para a caracterização da Hiperglicemia, adotou-se como referência a glicemia de jejum $\geq 126 \mathrm{mg} / \mathrm{d}^{13}$. Foram incluídos, também, os indivíduos diabéticos em tratamento atual com medicamentos, mesmo com a glicemia controlada.

A medida da pressão arterial (PA) foi efetuada duas vezes, após repouso de 10 minutos, com o mesmo aparelho (OMRON 705 CP), usando braçadeira adequada (Large). Para a caracterização da hipertensão arterial, foi adotado o critério de pressão arterial sistólica (PAS) $\geq 140 \mathrm{mmHg}$ e/ou pressão arterial diastólica $(\mathrm{PAD}) \geq 90 \mathrm{mmHg}^{14}$. A PA foi mensurada no braço direito posicionado à altura do coração, com o indivíduo relaxado na posição sentada. A PAS foi determinada ao aparecimento dos ruídos, fase I de Korotkoff e a PAD no desaparecimento dos ruídos, fase $\mathrm{V}$ de Korotkoff. Foram incluídos, também, os indivíduos hipertensos em tratamento atual com medicamentos, mesmo com PA controlada.

Os indivíduos classificados com presença de $\mathrm{CMCV}$ foram aqueles que apresentaram uma ou mais das variáveis, lipídeos plasmáticos, pressão arterial e glicemia fora das referências de normalidade, ou aqueles que estavam em uso de medicamentos. Os indivíduos classificados com ausência de CMCV foram aqueles que apresentaram as variáveis analisadas dentro dos valores de referência normais.

\section{Análise estatística}

Inicialmente, foi realizada estratificação para análise de modificação de efeito e confundimento. A análise para modificação de efeito foi realizada através da observação das medidas pontuais estratoespecíficas e seus intervalos de confiança. Foi utilizado intervalo de confiança de $95 \%$. A análise para confundimento foi feita comparando-se a odds ratio (OR) entre a associação bruta e ajustada pelos possíveis confundidores. Utilizou-se como parâmetro para identificar a diferença entre as associações o valor de 20\%. Logo após, foi realizada a análise através de regressão logística. A modelagem foi realizada com procedimento backward, partindo-se do modelo completo e retirando-se uma a uma as possíveis variáveis de confundimento que, quando suprimidas do modelo, causaram alteração igual ou superior a $20 \%$ na medida pontual de associação entre atividade física e CMCV. Finalmente, estimou-se a OR entre atividade física e CMCV através do modelo que melhor explicou esta associação.

Foram considerados como potenciais modificadores de efeito e confundimento da associação entre atividade física e CMCV as variáveis idade, etnia, estado civil e índice de massa corporal, todas introduzidas na modelagem de forma estratificada, exceto a idade que foi analisada de forma contínua. Para as demais variáveis, utilizou-se a seguinte estratificação: etnia $=1$ se branco, etnia $=2$ se pardo e etnia $=3$ se negra; estado civil $=1$ se solteiro, estado civil $=2$ se casado e estado civil $=3$ se viúvo; $\mathrm{IMC}=0$ se $<$ 40 e IMC $=1$ se $>40 ; \mathrm{CMCV}=0$ se apresentaram ausência das doenças metabólicas e $\mathrm{CMCV}=1$ se apresentaram presença de uma ou mais das doenças metabólicas analisadas; nível de atividade física $=$ 0 se classificado como ativo fisicamente e nível de atividade física $=1$ se classificado como inativo. 
Após análise para confundimento e modificação de efeito, a idade foi considerada como confundimento e a etnia, o estado civil e IMC como modificadores de efeito. Assim, o melhor modelo para explicar a associação entre atividade física e CMCV foi o ajustado por idade e estratificado por etnia, estado civil e IMC. Foi também analisado o efeito dose resposta do nível de atividade física para proteção das CMCV. Para esta análise, foram criadas variáveis DUMMIES para comparação entre o grupo de referência (inativos) e cada um dos outros extratos da variável atividade física. Foi utilizado o teste de Mantel Haenzel para testar a homogeneidade dos valores do OR entre os estratos de cada variável com nível de significância de 0,05. O intervalo de confiança foi estabelecido em $95 \%$. Empregou-se o programa estatístico "STATA" versão 7.0.

\section{RESULTADOS}

A caracterização da amostra pode ser observada na tabela 1 .

Na tabela 2, pode ser observado o nível de atividade física da população estudada e a prevalência das CMCV.

Pode-se observar que a maioria das mulheres estava inativa fisicamente. Já em relação às $\mathrm{CMCV}$, foi observado que a maioria das mulheres apresentou a presença das mesmas.

A tabela 3 demonstra a associação bruta e ajustada por idade entre a atividade física e $\mathrm{CMCV}$ em mulheres obesas.

A atividade física apresentou associação com as CMCV na associação bruta e quando ajustado por idade, demonstrando efeito protetor para as CMCV em mulheres obesas.

A tabela 4 demonstra a associação entre atividade física e CMCV ajustado por idade e estratificado por etnia, IMC e estado civil.

A atividade física apresentou associação com as CMCV quando estratificado por etnia, IMC e estado civil, exceto entre as viúvas.

A tabela 5 demonstra o efeito dose resposta entre o nível de atividade física CMCV.

Foi observado que quanto maior o nível de atividade física maior a proteção para as CMCV. As mulheres obesas classificadas como muito ativas fisicamente apresentaram maior proteção para a presença das $\mathrm{CMCV}$.

\section{DISCUSSÃO}

Os resultados do presente estudo demonstraram que
Tabela 1. Caracterização da amostra.

\begin{tabular}{|c|c|c|}
\hline Variável & $M \pm D P$ & IC (95\%) \\
\hline Idade (anos) & $\begin{array}{c}44,1 \pm 10,4 \\
(21-75)\end{array}$ & \\
\hline Peso (kg) & $\begin{array}{c}107,7 \pm 18,3 \\
(72-201)\end{array}$ & \\
\hline Estatura $(\mathrm{cm})$ & $\begin{array}{c}158 \pm 0,06 \\
(145-173)\end{array}$ & \\
\hline IMC ( $\left(\mathrm{kg} / \mathrm{m}^{2}\right)$ & $\begin{array}{c}42,79 \pm 6,32 \\
(31,2-70,5)\end{array}$ & \\
\hline $\begin{array}{c}\text { Etnia }(\%) \\
\text { Branco } \\
\text { Pardo } \\
\text { Negro }\end{array}$ & $\begin{array}{l}30,2 \\
45,9 \\
23,8\end{array}$ & $\begin{array}{l}(25,7-34,7) \\
(41,9-50,8) \\
(19,5-28,0)\end{array}$ \\
\hline $\begin{array}{c}\text { Estado civil (\%) } \\
\text { Solteiro } \\
\text { Casado } \\
\text { Viúvo }\end{array}$ & $\begin{array}{c}33,1 \\
60,5 \\
6,5\end{array}$ & $\begin{array}{c}(28,4-37,6) \\
(55,6-65,2) \\
(4,1-8,7)\end{array}$ \\
\hline
\end{tabular}

$M$ = média; $D P$ = desvio padrão; $I C$ = intervalo de confiança

Tabela 2. Classificação nível de atividade física e prevalência de comorbidades cardiovasculares.

\begin{tabular}{lcc}
\hline Variáveis & Percentual & IC $(95 \%)$ \\
\hline Atividade física & & \\
Ativo & 31,52 & $(28,1-34,9)$ \\
Inativo & 68,48 & $(63,8-73,0)$ \\
CMCV & & \\
Presença & 73,90 & $(69,6-78,2)$ \\
Ausência & 26,10 & $(21,8-30,4)$ \\
\hline
\end{tabular}

IC = intervalo de confiança; $\mathrm{CMCV}=$ comorbidades cardiovasculares.

Tabela 3. Associação bruta e ajustada por idade entre Atividade Física e Comorbidades cardiovasculares em mulheres obesas.

\begin{tabular}{lcc}
\hline Atividade Física & $\begin{array}{c}\text { Associação Bruta } \\
\text { OR (IC 95\%) }\end{array}$ & $\begin{array}{c}\text { Ajuste por idade } \\
\text { OR (IC 95\%) }\end{array}$ \\
\hline Inativos & 1,00 & 1,00 \\
Ativos & $0,11(0,04-0,24)$ & $0,11(0,04-0,25)$ \\
\hline
\end{tabular}

OR = Odds ratio; $I C$ = intervalo de confiança.

Tabela 4. Atividade Física e Comorbidades cardiovasculares ajustado por idade e estratificado por etnia, estado civil e IMC.

\begin{tabular}{lcrc}
\hline \multicolumn{1}{c}{ Variáveis } & OR & IC $(95 \%)$ & P \\
\hline Etnia & & & \\
Branca & 0,19 & $(0,05-0,71)$ & 0,10 \\
$\begin{array}{l}\text { Parda } \\
\text { Negra }\end{array}$ & 0,04 & $(0,01-0,20)$ & \\
& 0,16 & $(0,03-0,78)$ & \\
Estado civil & & & \\
Solteiro & 0,22 & $(0,07-0,63)$ & 0,04 \\
Casado & 0,04 & $(0,01-0,20)$ & \\
Viúvo & 1,01 & $(0,91-1,13)$ & \\
IMC & & & \\
$<40$ & 0,12 & $(0,03-0,43)$ & 0,70 \\
$>40$ & 0,09 & $(0,03-0,28)$ & \\
\hline
\end{tabular}

$\mathrm{OR}=$ Odds ratio; $\mathrm{IC}=$ intervalo de confiança. 
Tabela 5. Efeito dose resposta do nível de atividade física e comorbidades cardiovasculares em obesas.

\begin{tabular}{lcc}
\hline \multicolumn{1}{c}{ Nível de Atividade Física } & OR & IC $(95 \%)$ \\
\hline Inativos & 1,00 & \\
Insuficiente ativo & 0,90 & $(0,83-0,97)$ \\
Ativos & 0,65 & $(0,59-0,71)$ \\
Muito ativo & 0,50 & $(0,29-0,84)$ \\
\hline
\end{tabular}

OR = Odds ratio; IC = intervalo de confiança.

a atividade física promoveu proteção para as CMCV em mulheres obesas na associação bruta e quando ajustado por idade e estratificado por etnia, IMC e estado civil, exceto para viúvas. Foi evidenciado que a atividade física contribui para a ausência das $\mathrm{CMCV}$, independente dos valores atribuídos ao IMC, demonstrando que mulheres obesas que praticam atividades físicas regularmente diminuem o risco para o desenvolvimento das $\mathrm{CMCV}$.

Em estudo longitudinal ${ }^{15}$, realizado durante o período de 12 a 16 anos, com uma amostra de 1413 indivíduos, com faixa etária de 35 a 74 anos, demonstrou-se que um gasto calórico acima de 2000 kcal por semana, diminui significativamente os índices de mortalidade por doenças cardiovasculares. Nesse sentido, outros autores ${ }^{16}$ estudaram a associação entre atividade física e mortalidade em 25.433 mulheres, demonstrando que a atividade física apresentou uma associação inversa com a mortalidade.

No presente estudo, não foi realizado um acompanhamento longitudinal da população, podendo ser um fator limitante, já que foi analisada apenas a última semana de cada indivíduo em relação à prática de atividades físicas. A literatura tem recomendado ${ }^{17}$ que adultos para adquirir proteção para as $\mathrm{CMCV}$, devem acumular, pelo menos, trinta minutos de atividades físicas em intensidade moderada, na maioria dos dias da semana, com um gasto calórico de 200 calorias por dia.

Indivíduos com risco aumentado para o desenvolvimento da síndrome metabólica que praticam atividades físicas nos momentos de lazer, adquirem uma maior proteção para mesma, quando comparados a indivíduos que são sedentários nos momentos de lazer ${ }^{18}$. A atividade física está associada à proteção para o desenvolvimento do diabetes mellitus, bem como a redução dos riscos das doenças causadas em pacientes diabéticos. Estudo recente ${ }^{19}$ demonstrou que indivíduos que praticavam atividades físicas em moderada intensidade, apresentaram uma maior expectativa de vida e maior proteção para o diabetes mellitus quando comparados a indivíduos que praticavam atividades físicas em baixa intensidade. Já os indivíduos que praticavam atividades físicas em baixa intensidade, apresentaram uma maior expectativa de vida e maior proteção para o diabetes mellitus, quando comparados a indivíduos que eram sedentários.

Outros estudos recentes ${ }^{20,21}$ analisaram a relação entre obesidade, atividade física, doenças crônicas degenerativas e doenças cardiovasculares. Foi evidenciado que a atividade física e a obesidade apresentaram-se como variáveis independentes para o acometimento destas patologias, sendo demonstrada a importância da manutenção do IMC em níveis normais e da prática regular de atividades físicas para prevenção dos agravos metabólicos e cardiovasculares. Nessa linha de pensamento, tem sido demonstrado que a atividade física promove proteção para as CMCV em indivíduos com obesidade ${ }^{22}$.

No presente estudo, foi evidenciado que maiores níveis de atividades físicas promovem maior proteção para as CMCV. Foi observado que mulheres obesas que praticam atividades físicas de moderada a alta intensidade adquirem um maior efeito protetor para as CMCV. Os trabalhos têm demonstrado ${ }^{23}$ que indivíduos que praticam atividades físicas de moderada a alta intensidade (4 a 6 Mets e $\geq 6$ Mets, respectivamente) apresentaram uma maior proteção para a doença cardiovascular e maior longevidade. Estudo realizado ${ }^{24}$ com 90 obesos relatou que as atividades físicas de moderada a alta intensidade promovem uma maior proteção para a doença cardiovascular do que atividades físicas realizadas em baixa intensidade.

Nesse sentido, foi evidenciado ${ }^{25}$ que a prática de atividades físicas em moderada intensidade promove um maior efeito protetor para as co-morbidades cardiovasculares. Estudo realizado ${ }^{26}$ com 775 indivíduos, entre 45 e 70 anos, demonstrou que o gasto energético está associado com redução da PA. Indivíduos com um maior gasto calórico apresentam menores níveis de pressão arterial, independente da obesidade e da capacidade cardiorrespiatória

Outro recente estudo ${ }^{27}$, que procurou analisar a associação entre IMC, aptidão cardiorrespiratória e síndrome metabólica em 200 mulheres com faixa etária entre 18 e 40 anos, demonstrou que o IMC, a aptidão cardiorrespiratória e o nível de atividade física são variáveis importantes para reduzir a prevalência e incidência da síndrome metabólica.

\section{CONCLUSÃO}

Na população estudada, a maioria das mulheres apresentou-se com presença das CMCV e foram 
classificadas como inativas fisicamente. Quando analisada a associação entre atividade física e CMCV, tanto bruta quanto ajustada por idade e estratificada por etnia, estado civil e IMC foi evidenciado que a atividade física apresentou-se como uma variável protetora para as $\mathrm{CMCV}$. Ao se analisar o efeito dose resposta, foi evidenciado que maiores níveis de atividades físicas promovem maior proteção para as CMCV. Foi demonstrado, no presente estudo, que mulheres obesas classificadas como muito ativas fisicamente apresentaram maior proteção para as CMCV. Portanto, a atividade física deve ser sugerida para mulheres obesas, visando afastar o risco para $\mathrm{CMCV}$, uma vez que a obesidade já se apresenta como um fator de risco importante para estas doenças. Aumentos gradativos no nível de atividade física também devem ser sugeridos para esta população, visando proporcionar uma maior proteção para a ausência das CMCV. A adoção de um estilo de vida ativo fisicamente deve fazer parte da rotina diária dos indivíduos com obesidade, buscando uma maior qualidade de vida e longevidade.

\section{REFERÊNCIAS BILBIOGRÁFICAS}

1. Kelly T, Yang W, Chen CS, Reynolds K, He J. Global burden of obesity in 2005 and projections to 2030. Int J Obes 2008;32(9):1431-7.

2. Instituto Brasileiro de Geografia e Estatística. Análise da disponibilidade domiciliar de alimentos e do estado nutricional no Brasil 2002-2003. Rio de Janeiro: IBGE; 2004.

3. Hubert HB, Feinleid M, McNamara PM, Castel WP. Obesity as an independent risk factor for cardiovascular disease: a 26-year follow-up of participants in the Framingham Heart Study. Circulation 1983;67(5):968-77.

4. Harris TB, Ballard-Barbacs R, Madans J, Makuc DM, Feldman JJ. Overweight, weight loss, and risk of coronary heart disease in older women. The NHANES I Epidemiologic Follow-up Study. Am J Epidemiol 1993;137(12):1318-27.

5. Paschoal MA, Polessi EA, Simioni FC. Avaliação da variabilidade da freqüência cardíaca em mulheres climatéricas treinadas e sedentárias. Arq Bras Cardiol 2008;90(2):80-6.

6. Erlichman J, Kerbey AL, James WP. Physical activity and its impact on health outcomes. Paper 1: The impact of physical activity on cardiovascular disease and all-cause mortality: an historical perspective. Obes Rev 2002;3(4):257-71.

7. Blair SN, Harold WK, Paffenbarger RS, Clark DG, Cooper KH, Gibbons MD. Physical Fitness and allCause Mortality a Prospective Study of Healthy Men and Women. JAMA 1989;262:2395-401.

8. Giacomini, M.C.C.; Santos, A.L.P.; Giacomini, O. Comparação das Analises Bioquímicas em Mulheres Obesas Submetidas a um Programa de Treinamento Físico Aeróbico. Efdeportes.com. 2007.
9. Brochu M, Tchernof A, Dionne, IJ, Sites, CK, Eltabbakh GH, Sims EAH, et al. What are physical characteristics associated with a normal metobolic profile despite a high level of obesity in postmenopausa women? J Clin Endocrinol Metab 2001;86(3):1020-5.

10. World Health Organization. Obesity. Preventing and Managing the Global Epidemic. Report of a WHO Consultation on Obesity. Geneva: World Health Organization, 1998.

11. Matsudo SM, Araújo TL, Matsudo VKR, Andrade DR, Andrade EL, Oliveira LC, Braggion G. Questionário Internacional de Atividade Física (IPAQ): estudo de validade e reprodutibilidade no Brasil. Rev Bras Ativ Saúde 2001;6(2):5-18.

12. Sociedade Brasileira de Cardiologia. $2^{\mathrm{a}}$ Consenso Brasileiro sobre Dislipidemias. Arq Bras Cardiol 1996;67(2):1-15.

13. Sociedade Brasileira de Diabetes. Consenso Brasileiro de Diabetes: Diagnóstico e classificação do diabetes melito e tratamento do diabetes melito do tipo 2. Rio de Janeiro: Diagraphic Editora, 2003

14. JVC VII - Joint National Committee on Detection Evaluation, and Treatment of High Blood Pressure. The Fifth Report of the Joint National Committee on Detection, Evaluation, and Treatment of High Blood Pressure. Arch Intern Med 1993;153:154-83.

15. Paffenbarger RS, Hyde RT, Wing AL, Hsieh CC. Physical Activity, all-cause mortality, and longevity of College Alumni. N Engl J Med 1986, 314(10):605-13.

16. Blair SN, Mccloy CH. Research Lecture: Physical Activity, Physical Fitness and Health. Res Quart Exerc Sport 1993;64(4):365:76.

17. CDC \& ACSM. Physical Activity and Public Health. A recommendation from the centers for disease control and prevention and the American college of sports medicine. JAMA 1995; 273:402-7.

18. Laaksonen DE, Lakka HM, Salonen JT, Niskanen LK, Rauramaa R, Lakkata. Low Levels of Leisure-Time Physical Activity and Cardiorespiratory Fitness Predict Development of the Metabolic Syndrome. Diabetes Care 2002;25:1612-18.

19. Jonker JT, De Laet C, Franco OH, Peeters A, Mackenbach J, Nusselder WJ. Physical activity and life expectancy with and without diabetes: Live table analysis of the Framingham Heart Study. Diabetes Care 2006;29: 38-43.

20. Lity, Rana JS, Manson JE, Willet WC, Stampfer MJ, Colditz GA, Rexrode KM, Hu FB. Obesity as compared with Physical Activity in Predicting Risk of Coronary Heart Disease in Women. Circulation 2006; 113(4):449-506.

21. Rana JS, Litty, Manson JE, Hu FB. Adiposity Compared with Physical Inactivity and Risk of Type 2 Diabetes in Women. Diabetes Care 2007; 30(1);53-8.

22. Marchesini G, Pontiroli A, Salvioli G, Novi RF, Vitacolonna E, Taboga C, Ciccarone AM, Crossi E, Quavadis Study Group. Snoring, Hipertension and Type 2 Diabetes in Obesity. Protection by Physical Activity. J Endocrinol Invest 2004;27(2):150-7. 
23. Lee IM, Sesso HD, Paffenbarger RS. Physical activity and coronary heart disease risk in men: does the duration of exercise episodes predict risk. Circulation 2000;102:981-6.

24. Okura T, Nakata Y, Tanaka K. Effects of exercise intensity on physical fitness and risk factor for coronary heart disease. Obes Res 2003;11(9): 1131-9.

25. Jakicic J M, Otto A D. Physical Activity Considerations for the treatment and prevention of Obesity. Am J Clin Nutr 2005; 82:226-29.

26. Wareham NJ, Wong MY, Hennings S, Mitchell J, Kirsten R, Kennedy C, Nicholas ED. Quantifying the associantion between habitual energy expenditure and blood pressure. Int J Epidemiol 2000; 29:655-60.

27. Thompson JL, Herman CJ, Allen P, Helitzer DL, Wilson ND, Whyte AN, Perez GE, Wolfe VK. Associations Beteween Body Mass Index, Cardiorespiratory Fitness, Metabolic Syndrome and Impaired Fasting Glucose in Young, Urban Native American Women. Metab Syndr Relat Disord;2007;5(1):45-54.
Endereço para correspondência

Cristiano Penas Seara Pitanga

Rua Rodolfo Coelho Cavalcante, n 196, Edf.

Colinas do Atlântico

Bairro: Jardim Armação, Salvador.

E-mail: cpspitanga@lognet.com.br 